\title{
Dual Fluorescence in Glutathione-Derived Carbon Dots Revisited
}

Yadolah Ganjkhanlou, ${ }^{*}$ J.J. Erik Maris, ${ }^{*}$ Joris Koek, Romy Riemersma, Bert M. Weckhuysen,

and Florian Meirer ${ }^{*}$

Inorganic Chemistry and Catalysis, Debye Institute for Nanomaterials Science, Utrecht

University, 3584 CG Utrecht, The Netherlands

* Corresponding author e-mail address: f.meirer@uu.nl 
Table S1. Details of the synthesis reagents and the solvothermal treatment temperature, time, and additives.

\begin{tabular}{|c|c|c|c|c|c|c|}
\hline Sample & GSH (g) & Solvent & Solvent (mL) & $\mathrm{T}\left({ }^{\circ} \mathrm{C}\right)$ & Time & Additives \\
\hline GSH-FA-3h & 0.3 & FA & 10 & 180 & $3 \mathrm{~h}$ & -------------- \\
\hline GSH-FA-18h & 0.3 & FA & 10 & 180 & $18 \mathrm{~h}$ & ------------- \\
\hline GSH-1-FA-3h & 0.15 & FA & 10 & 180 & $3 \mathrm{~h}$ & -------------- \\
\hline GSH-h-FA-3h & 1.0 & FA & 10 & 180 & $3 \mathrm{~h}$ & ------------- \\
\hline GSH-DMF-3h & 0.3 & DMF & 10 & 180 & $3 h$ & -------------- \\
\hline GSH-H2O-3h & 0.3 & $\mathrm{H}_{2} \mathrm{O}$ & 10 & 180 & $3 \mathrm{~h}$ & -------------- \\
\hline $\begin{array}{l}\text { GSH-FA-5min- } \\
\text { Acidic }\end{array}$ & 0.1 & FA & 5 & 180 & $5 \mathrm{~min}$ & $\begin{array}{l}1 \quad \mathrm{~mL} \\
\mathrm{HCl}(37 \%)\end{array}$ \\
\hline GSH-FA-5min & 0.1 & FA & 5 & 180 & $5 \mathrm{~min}$ & --------------- \\
\hline $\begin{array}{l}\text { GSH-FA-5min- } \\
\text { Basic }\end{array}$ & 0.1 & FA & 5 & 180 & $5 \mathrm{~min}$ & $0.2 \mathrm{~g} \mathrm{NaOH}$ \\
\hline
\end{tabular}


Table S2. Tentative assignment of observed IR bands.

\begin{tabular}{|c|c|c|}
\hline Band position $\mathrm{cm}^{-1}$ & Possible assignment & Description and Ref. \\
\hline$>3300$ & $\mathrm{O}-\mathrm{H}$ stretching & Broad band due to $\mathrm{H}$ bonding, ${ }^{1-3}$ \\
\hline 3320 & $\mathrm{~N}-\mathrm{H}$ stretching & $1-3$ \\
\hline 3185 & C-H stretching & $1-3$ \\
\hline 2900 & $\mathrm{C}-\mathrm{H}$ stretching & $1-3$ \\
\hline 2885 & C-H stretching & $1-3$ \\
\hline 1673 & $\mathrm{C}=\mathrm{O}$ or $\mathrm{C}=\mathrm{N}$ stretching & $1-3$ \\
\hline 1591 & $\mathrm{C}=\mathrm{C}$ stretching & $1-3$ \\
\hline 1387 & C-H bending & $1-3$ \\
\hline 1309 & C-N stretching & In aromatic amines, ${ }^{1-3}$ \\
\hline 1098 & C-O stretching & $1-3$ \\
\hline 1044 & $\mathrm{~S}=\mathrm{O}$ & 1 \\
\hline 950 & $\mathrm{C}=\mathrm{C}$ bending & $1-3$ \\
\hline 795 & $\mathrm{C}-\mathrm{H}$ bending & $1-3$ \\
\hline
\end{tabular}



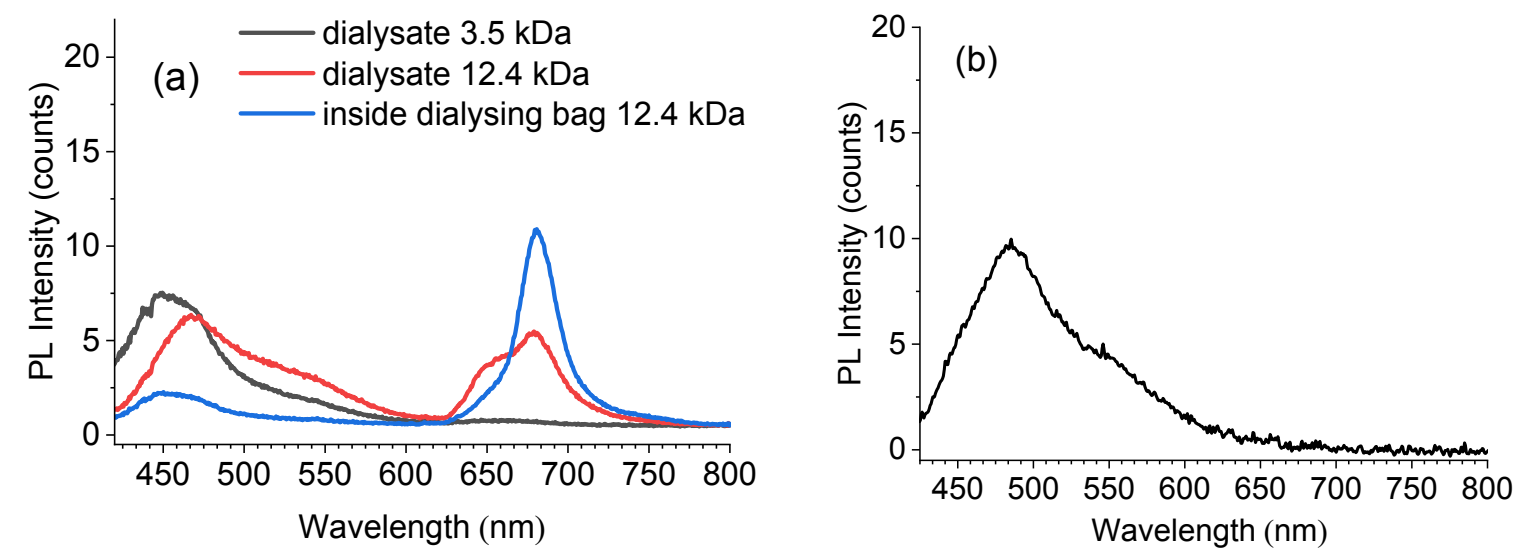

Figure S1. a) Photoluminescence emission spectrum of dialysate passed through a $3.5 \mathrm{kDa}$ and 12.4 kDa membrane and products inside a $12.4 \mathrm{kDa}$ membrane of GSH-FA-3h dialysed for a week.

b) Photoluminescence emission spectrum of a glutathione and formamide solution heated at $\mathrm{T}=$ $160^{\circ} \mathrm{C}$ for $1 \mathrm{~min}$. 


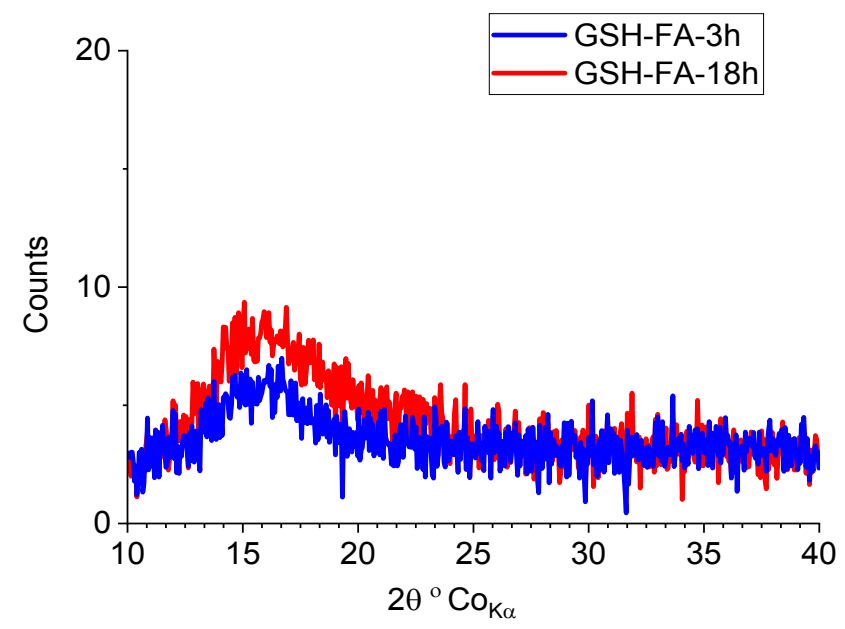

Figure S2. The X-ray diffraction pattern of sample GSH-FA-3h and GSH-FA-18h after dialysis with $3.5 \mathrm{kDa}$ membrane and deposition on glass cover slip.

The X-ray diffraction patterns of the prepared carbon dots have a broad diffraction line at $2 \theta C o K \alpha$ of $16^{\circ}(d=5.72 \AA)$. Carbon dots synthesized with bottom-up approaches (especially those synthesized below $200^{\circ} \mathrm{C}$ ) usually are near-amorphous in nature ${ }^{4}$, which results in broadening of the diffraction line. The observed $d$ spacing of $5.72 \AA$ is larger than the typical 002-diffraction line of graphene (observed at $d \approx 3.38 \AA$ ). Enlarged interlayer spacing of CDs is reported to be due to the presence of different functional groups in CDs (especially oxygen-containing ones). ${ }^{5}$ 


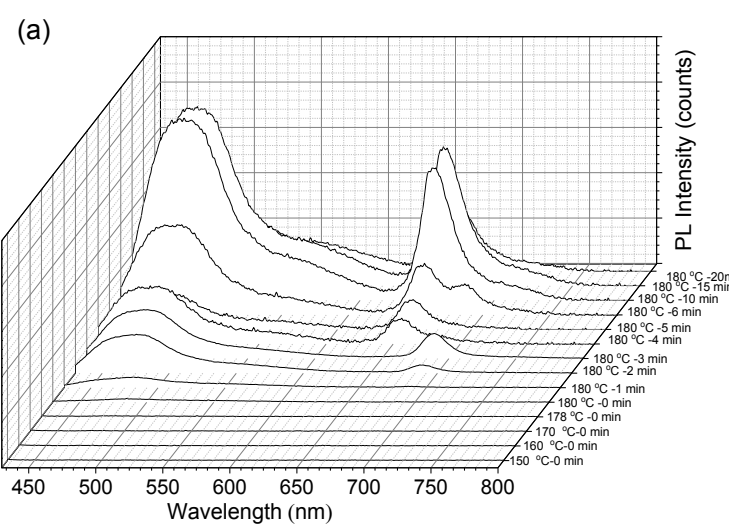

(b)

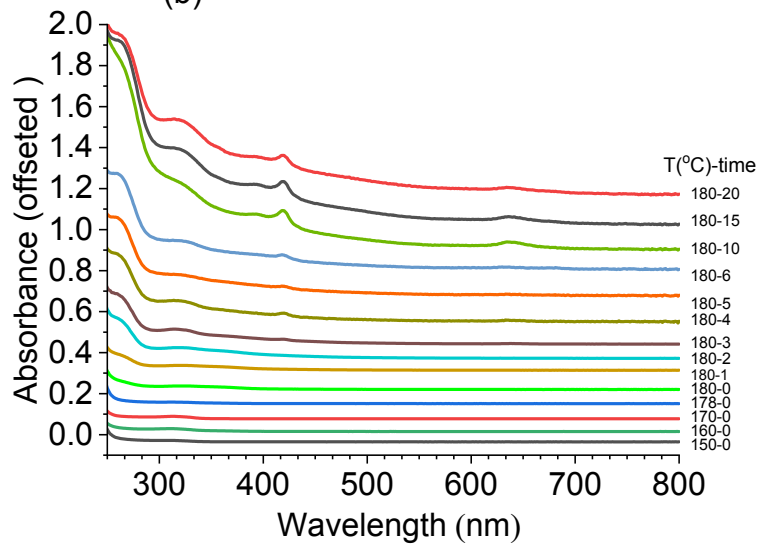

Figure S3. In-situ a) photoluminescence (PL) emission and b) offset UV-Vis absorbance spectra of $100 \mathrm{mg}$ glutathione in $5 \mathrm{ml}$ formamide solution during the heating up to $180^{\circ} \mathrm{C}$ and hold at this temperature sampled at various times. The formation of porphyrin occurs mostly in the first 10 minutes of the reaction, after the onset of the formation of blue-emitting fluorophores. 


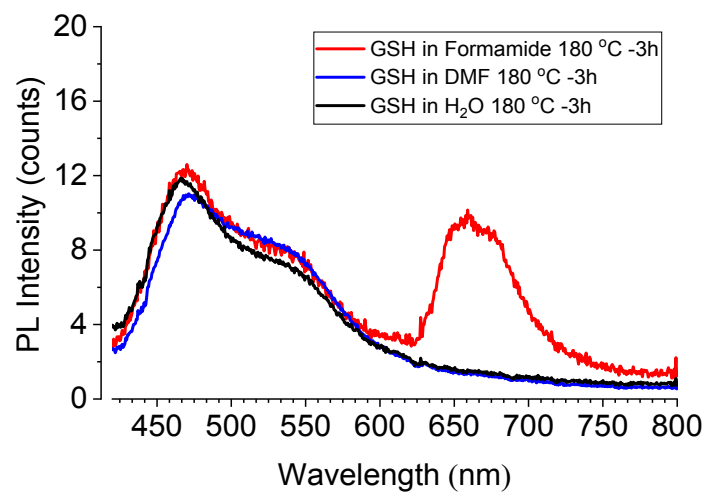

Figure S4. Photoluminescence emission spectrum of reaction products of the solvothermal treatment of glutathione in different solvents. Only glutathione in formamide yields photoluminescence emission in the $600-750 \mathrm{~nm}$ regime. 
(a)

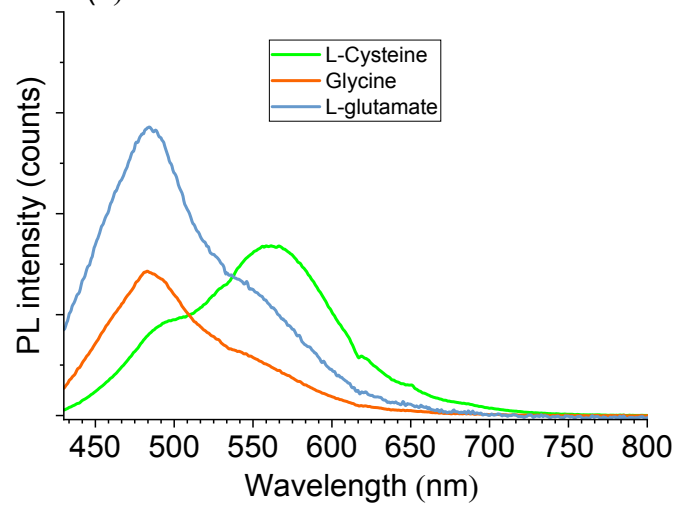

(b)

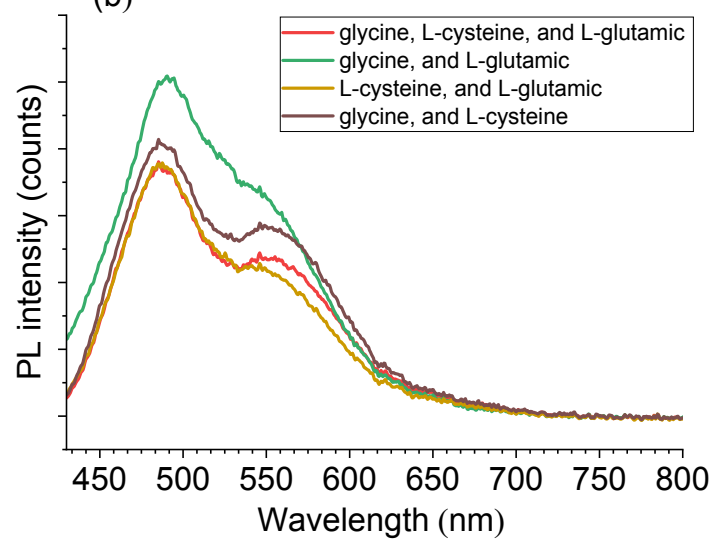

Figure S5. a-b) Photoluminescence emission spectrum of the reaction products of the solvothermal treatment in formamide of single (a) and pairs/triplets of (b) amino acids in glutathione. Even though these amino acids make up the glutathione peptide, they do not yield emission in the 600$750 \mathrm{~nm}$ regime, indicating that no porphyrin is formed. The concentration of all reagents in formamide was $150 \mathrm{mM}$ in this experiment. 

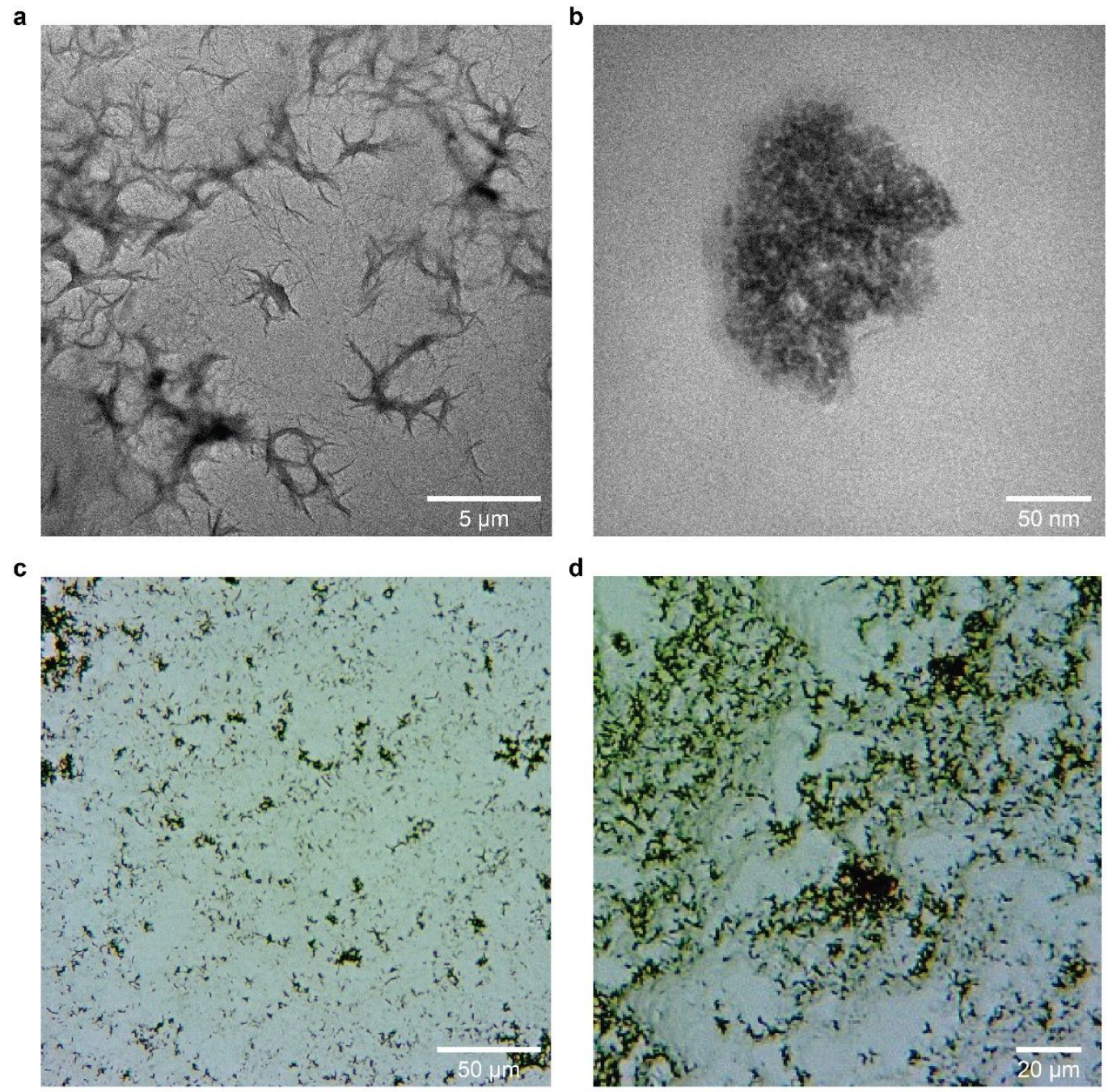

Figure S6. a-b) Transmission electron microscopy (TEM) images of GSH-FA-3h with selfassembled porphyrin (a) and carbon dot clusters (b) dried on a TEM grid. The carbon dots are visible at high magnification. The size of the carbon dots is around $3-5 \mathrm{~nm}$, which is in agreement with AFM topography measurements (Fig. 3d). c-d) Optical microscopy images of dendritic selfassembly of porphyrin observed in a dried GSH-FA-3h sample. The green color of dendritic structures is in line with the measured UV-Vis absorption spectrum of the porphyrin derivatives, 
which verifies that these self-assemblies are related to porphyrin. Indeed, porphyrin is a two-

dimensional macromolecule, and a majority of its derivatives are hard to crystallize and tend to

form amorphous assemblies. ${ }^{6-7}$ 


\section{REFERENCES}

1. Lin-Vien, D.; Colthup, N. B.; Fateley, W. G.; Grasselli, J. G., The Handbook of Infrared and Raman Characteristic Frequencies of Organic Molecules, Elsevier, 1991.

2. Mitchell, R. T., Synthesis of Water Soluble Porphyrins and Their Applications. 2016.

3. Namitha, P. P.; Saji, A.; Francis, S.; Rajith, L., Water Soluble Porphyrin for the Fluorescent

Determination of Cadmium Ions. Journal of fluorescence 2020, 30, 527-535.

4. Xiong, Y.; Schneider, J.; Ushakova, E. V.; Rogach, A. L., Influence of Molecular Fluorophores on the Research Field of Chemically Synthesized Carbon Dots. Nano Today 2018,

23, 124-139.

5. De, B.; Karak, N., A Green and Facile Approach for the Synthesis of Water Soluble Fluorescent Carbon Dots from Banana Juice. Rsc Advances 2013, 3, 8286-8290.

6. Battersby, A. R., Tetrapyrroles: The Pigments of Life. Natural product reports 2000, 17, 507-526.

7. Goldberg, I., Crystal Engineering of Nanoporous Architectures and Chiral Porphyrin Assemblies. CrystEngComm 2008, 10, 637-645. 
